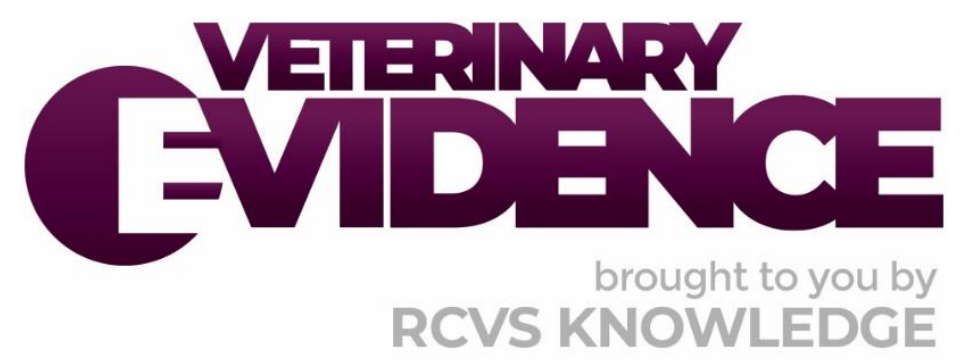

\title{
Comparison of epidural morphine and buprenorphine for hindlimb orthopaedic surgery in dogs
}

\author{
A Knowledge Summary by
}

Thomas Towers MA VetMB CertAVP(VA) MRCVS ${ }^{1 *}$

\footnotetext{
${ }^{1}$ Swayne \& Partners Ltd., The Veterinary Surgery, 84 Hamlet Road, Haverhill, Suffolk, CB9 8QQ

*Corresponding Author (tom.towers@cantab.net)
}

ISSN: 2396-9776

Published: 09 Jun 2020

in: Vol 5, Issue 2

DOI: 10.18849/VE.V5I2.291

Reviewed by: Andy Morris (BSc(Hons) BVSc CertAVP(GSAS)

MRCVS) and Tristan Merlin (DVM MSc

MVetMed)

Next Review Date: 14 Jul 2021 
KNOWLEDGE SUMMARY

\section{PICO question}

In dogs undergoing hindlimb orthopaedic surgery does epidural with local anaesthetic and buprenorphine provide equivalent intra- and postoperative analgesia as epidural with local anaesthetic and morphine?

\section{Clinical bottom line}

\section{Category of research question}

Treatment

The number and type of study designs reviewed

One double-blinded randomised controlled trial

\section{Strength of evidence}

Weak

\section{Outcomes reported}

Epidural analgesia with buprenorphine and bupivacaine may provide equivalent analgesia to more traditional morphine and bupivacaine epidural injection

\section{Conclusion}

There is weak evidence that buprenorphine may provide equivalent analgesia to morphine when combined with bupivacaine epidurally. The reduced regulatory requirements imposed on buprenorphine may sway some clinicians to utilise buprenorphine but further, higher powered, controlled trials are necessary to confirm equivalency

\section{How to apply this evidence in practice}

The application of evidence into practice should take into account multiple factors, not limited to: individual clinical expertise, patient's circumstances and owners' values, country, location or clinic where you work, the individual case in front of you, the availability of therapies and resources.

Knowledge Summaries are a resource to help reinforce or inform decision making. They do not override the responsibility or judgement of the practitioner to do what is best for the animal in their care. 


\section{Clinical Scenario}

Bessie is an adult female Border Collie with a complete rupture to the right cranial cruciate ligament. You are considering a tibial plateau leveling osteotomy (TPLO) to manage this injury and routinely use preservativefree morphine and bupivacaine epidurally as part of your analgesic management. Morphine is not licensed for use in dogs and is a controlled drug (Schedule 2) requiring strict record keeping and storage due to its abuse potential. Buprenorphine is available preservative-free in the UK and is licensed for use in dogs (although not via the epidural route), with a reduced abuse potential meaning, although it is controlled under Schedule 3 it is subject to less stringent controls on record keeping. You wonder whether buprenorphine would be an appropriate substitution, in terms of its analgesic efficacy, duration of action and adverse effects, for morphine in the epidural.

\section{The evidence}

The only study identified that was relevant to the PICO question was a randomised controlled trial by Smith \& $\mathrm{Yu}$ (2001) that directly compares the use of sole agent buprenorphine against morphine for epidural administration during surgical correction of cranial cruciate ligament rupture. Other papers identified compared various nerve block techniques to morphine or morphine-bupivacaine epidural and, as such, were not relevant to the question posed here.

Smith \& Yu (2001) performed epidural injections on 20 client-owned dogs from an unknown population, splitting them equally by an undisclosed method between receiving either $0.1 \mathrm{mg} \mathrm{kg}^{-1}$ morphine or $4 \mathrm{\mu g} \mathrm{kg}^{-1}$ buprenorphine diluted in saline $\left(0.2 \mathrm{~mL} \mathrm{~kg}^{-1}\right)$ to equal the volume of morphine. Cranial cruciate rupture was confirmed with radiography at least 16 hours prior to surgery under sedation with acepromazine $0.05 \mathrm{mg} \mathrm{kg}^{-1}$ and butorphanol $0.2 \mathrm{mg} \mathrm{kg}^{-1}$. On the day of surgery, premedication was achieved with acepromazine $0.1 \mathrm{mg}$ $\mathrm{kg}^{-1}$ followed by induction with propofol to effect $\left(4-6 \mathrm{mg} \mathrm{kg}^{-1}\right)$ and maintenance with halothane in oxygen via an endotracheal tube. The surgical technique employed for stabilisation of the cruciate ligament was lateral imbrication with monofilament suture.

Intraoperative monitoring included end-tidal halothane concentrations, heart rate, respiratory rate, noninvasive oscillometric blood pressure and end-tidal carbon dioxide measurement.

Postoperatively non-validated objective and subjective pain scores were utilised to determine when rescue analgesia may be required (intramuscular morphine, $1 \mathrm{mg} \mathrm{kg}^{-1}$ ) and if this was required the dog was considered a treatment failure and removed from further analysis.

The authors found no statistically significant difference between either group and concluded that buprenorphine epidural is as effective as morphine for analgesia during surgery to stabilise the cranial cruciate ligament, although the level of statistical significance utilised is not stated. The authors note that no adverse effects could be attributed to either epidural protocol.

However, the findings of this study must be interpreted in the light of the low post-hoc power calculation suggesting a high risk of type II statistical error, with a less than $10 \%$ chance of detecting statistically significant differences to a level of $\mathrm{P}=0.05$.

This study could be strengthened by formal assessment of the quality of anaesthesia (for example physiological indicators of nociception). Although ethical considerations limit the application, a control group of saline epidural medication would help to determine if buprenorphine is truly equivalent to morphine. 


\begin{tabular}{|c|c|}
\hline Population: & $\begin{array}{l}\text { Client-owned dogs with naturally occurring cranial cruciate ligament } \\
\text { rupture admitted for surgical repair }\end{array}$ \\
\hline Sample size: & 20 dogs \\
\hline Intervention details: & $\begin{array}{l}\text { - Premedication with acepromazine }\left(0.1 \mathrm{mg} \mathrm{kg}^{-1}\right) 20 \text { minutes } \\
\text { prior to induction of anaesthesia with } 4-6 \mathrm{mg} \mathrm{kg}^{-1} \text { propofol } \\
\text { and controlled or spontaneous ventilation. } \\
\text { - Random allocation equally between two epidural protocols, } \\
\text { each diluted with sterile saline to a total volume of } 0.2 \mathrm{~mL} \\
\mathrm{~kg}^{-1} \text {, administered immediately after induction of } \\
\text { anaesthesia: } \\
\circ 4 \mu \mathrm{kg}^{-1} \text { buprenorphine, or } \\
\circ 0.1 \mathrm{mg} \mathrm{kg}^{-1} \text { morphine. }\end{array}$ \\
\hline
\end{tabular}

- Stabilisation of the cranial cruciate ligament by extracapsular imbrication using monofilament suture.

\begin{tabular}{|c|c|}
\hline Study design: & Double-blinded randomised controlled trial \\
\hline Outcome studied: & $\begin{array}{l}\text { - Subjective measurement of postoperative pain using a non- } \\
\text { validated behavioural clinical instrument. } \\
\text { Objective postoperative pain assessment with a non- } \\
\text { validated physiological clinical instrument (heart rate, } \\
\text { respiratory rate, blood pressure). }\end{array}$ \\
\hline $\begin{array}{l}\text { Main findings: } \\
\text { (relevant to PICO question): }\end{array}$ & $\begin{array}{l}\text { - No statistically significant difference in objective or } \\
\text { subjective pain scores. } \\
\text { - Epidural buprenorphine at } 4 \mu \mathrm{gg}^{-1} \text { appears to provide } \\
\text { equivalent postoperative analgesia to epidural morphine at } \\
0.1 \mathrm{mg} \mathrm{kg}^{-1} \text {. } \\
\text { - There appears to be no adverse effects to either medication } \\
\text { protocol. }\end{array}$ \\
\hline Limitations: & $\begin{array}{l}\text { - Small sample size and post-hoc power calculation reveals a } \\
\text { low probability of detecting a true difference between the } \\
\text { two treatment arms. } \\
\text { - The level of statistical significance utilised is not stated. } \\
\text { - There is no control group (although ethical considerations } \\
\text { limit application). } \\
\text { - Intraoperative effects of the protocols are not formally } \\
\text { measured. Where attempts are made there are significant } \\
\text { issues with the data: } \\
\text { End-tidal } \mathrm{CO}_{2} \text { analysis is confounded by a subset of } \\
\text { dogs in each study arm being mechanically } \\
\text { ventilated. Ventilation should reduce the difference } \\
\text { between groups. }\end{array}$ \\
\hline
\end{tabular}




\begin{tabular}{|l|l|}
\hline $\begin{array}{c}\text { End-tidal halothane tension is }>1 \text { \%, higher than } \\
\text { previously determined minimum alveolar } \\
\text { concentrations (MAC) which is surprising when used } \\
\text { in combination with acepromazine and epidural } \\
\text { morphine, which have been shown to be MAC- } \\
\text { sparing. }\end{array}$ \\
$\begin{array}{l}\text { Dose of buprenorphine chosen is based on human data, } \\
\text { canine data is seemingly lacking, and significantly lower than } \\
\text { the licensed parenteral dose in the UK. } \\
\text { The extent of systemic absorption of buprenorphine by the } \\
\text { epidural route is not known in dogs and this study did not } \\
\text { make provisions to account for this possibility. } \\
\text { Not comparable to normal clinical practice as most } \\
\text { orthopaedic procedures will receive analgesic premedicants } \\
\text { (such as full } \mu \text { agonist opioids). } \\
\text { The pain scales used have not been validated (although it is } \\
\text { likely none were available in 2001). } \\
\text { This is an old study from 2001, anaesthetic and surgical } \\
\text { practices may have changed since. } \\
\text { Halothane is not commonly used in the UK at this } \\
\text { time. } \\
\text { ACP is rarely used as a sole premedicant and is } \\
\text { therefore often given at doses much less than } 0.1 \\
\text { mg kg-1. }\end{array}$ \\
Extracapsular prostheses are not commonly employed for \\
large dogs in the UK, with osteotomies preferred.
\end{tabular}

\section{Appraisal, application and reflection}

There is a dearth of high-quality evidence to compare the treatments in this PICO. Although the paper by Smith \& Yu (2001) is a randomised controlled trial, sitting high in the hierarchy of evidence, it has some flaws that mean application to day-to-day clinical practice may be challenging.

Most significantly, the paper presented has a low power; post-hoc power calculations suggest that to give a 20 $\%$ chance of detecting a statistically significant difference in pain scores over the first 2 hours, to $P=0.05$, a sample size of 69 dogs per group is required - much larger than the 10 dogs per group utilised in the study. Ideally, such sample size calculations should have been performed prior to commencement, or after a brief pilot study.

The pain score used during the study is not validated, although the current validated pain scoring systems (such as the Glasgow Composite Measure Pain Scale - Short Form (Reid et al., 2007)) were not available in 2001. As the systems are not validated, they may not be best designed for the detection of pain or may demonstrate observer bias and reduce the reproducibility of this study; further, the effect size (significant difference in scores) chosen of 0.5 pain score units may not reflect a clinically significant difference in analgesia and the authors do not address the confounding sedation due to acepromazine dosing. 
A further flaw is the lack of knowledge of the extent of systemic absorption of epidural buprenorphine; Smith \& Yu (2001) provide a brief literature review in this regard and summarise good evidence for the lack of absorption for morphine but limited data for buprenorphine in dogs. The author of this Knowledge Summary was also unable to find published studies on the bioavailability of epidural buprenorphine in dogs. Therefore, it cannot be ruled out that the treatment effect seen for buprenorphine is attributable to a systemic effect. Should this be the case, the higher risk of epidural drug administration would preclude this route of administration in patients in favour of other parenteral routes.

Although an attempt is made to compare the end-tidal $\mathrm{CO}_{2}$ tensions between groups, the methodology states that an unspecified proportion of dogs in each study arm were mechanically ventilated. Ventilation would be expected to reduce differences between groups so must be taken into account when interpreting this data. The requirement for ventilation may be due to blunting of physiological ventilatory responses caused by halothane, which in this study is seen at mean \pm SD end-tidal concentrations (\%) of $1.04 \pm 0.26$ (buprenorphine) and $1.06 \pm 0.18$ (morphine). These end-tidal halothane tensions are higher than previously reported minimum alveolar concentrations (MAC) for halothane with $0.1 \mathrm{mg} \mathrm{kg}^{-1}$ epidural morphine (as in (Smith \& Yu, 2001)) of $0.6 \pm 0.017$ (Valverde et al., 1989) and despite the use of large doses of acepromazine as a premedicant, which has been shown to be MAC sparing, producing end-tidal concentrations of $0.58 \pm 0.044$ with acepromazine doses of $0.1 \mathrm{mg} \mathrm{kg}^{-1}$ in one study (Heard et al., 1986).

Additional to these clinical concerns regarding end-tidal $\mathrm{CO}_{2}$ and halothane tensions, the statistical handling of both variables is unclear. The data sets for each variable would consist of a series of numbers for every dog in both study arms but the ANOVA analysis applied requires one data point from each dog; the authors do not elaborate on the methodology used to derive that number. As anaesthetic and ventilatory requirements may be expected to vary over time in response to surgical stimulus, this statistical approach is unlikely to be appropriate for these data.

In terms of application to clinical practice, there are some deviations that may be significant. No analgesic medication was administered to these patients other than the treatments being studied, which contrasts current anaesthetic practice where systemic opioid analgesics are often employed (Epstein et al., 2015). The $50 \%$ requirement for rescue analgesia in both trial arms may reflect inadequate perioperative analgesia.

Current surgical preferences may lie towards more invasive osteotomy techniques (such as tibial tuberosity transposition or TPLO) rather than extracapsular imbrication (Bergh et al., 2014) so it is feasible that the analgesic requirements of different surgical techniques will differ.

Unfortunately, the only paper obtained from the literature search did not utilise local anaesthetic in the epidural protocols tested and is therefore not completely relevant to the PICO question posed.

Therefore, it is not possible to make a recommendation to utilise buprenorphine epidurally in hindlimb orthopaedic surgery in dogs over morphine, although the reduced abuse potential and regulatory burden may make this attractive to individual practitioners. Further randomised controlled trials are needed to answer this PICO question. 


\begin{tabular}{|c|c|}
\hline \multicolumn{2}{|l|}{ Search Strategy } \\
\hline $\begin{array}{r}\text { Databases searched and dates } \\
\text { covered: }\end{array}$ & $\begin{array}{l}\text { CAB Abstracts on VCAB Direct } 1973 \text { to July 12, } 2019 \text { (week } 28 \text { 2019) } \\
\text { Medline } 1946 \text { to July 12, } 2019 \text { via Ovid (week } 28 \text { 2019) }\end{array}$ \\
\hline Search terms: & $\begin{array}{l}\text { CAB Abstracts and Medline: } \\
\text { (dog OR dogs OR canis OR canine OR canid*) AND (stifle OR joint OR } \\
\text { joints OR limb OR limbs OR "hind limb" OR hind-limb OR hindlimb } \\
\text { OR tarsus OR tarsal OR hock OR hocks OR ankle* OR patella* OR } \\
\text { knee* OR phalanx OR phalanges OR orthopaedic* OR orthopedic* } \\
\text { OR fracture*) AND (morphine OR buprenorphine) AND (epidural* } \\
\text { OR extradural* OR "extra dural*" OR extra-dural* OR extrathecal* } \\
\text { OR "extra thecal*" OR extra-thecal* OR "conduction anaesthesia" } \\
\text { OR "conduction anesthesia") }\end{array}$ \\
\hline Dates searches performed: & $14 / 07 / 2019$ \\
\hline
\end{tabular}

\section{Exclusion / Inclusion Criteria}

Exclusion: Irrelevant to PICO, review articles, not available in English, book chapters or sections, other non-peer reviewed material, articles not available for review, duplicated articles

Inclusion: Peer-reviewed material including case series, observational or interventional studies and systematic reviews relating to the use of buprenorphine or morphine epidurally in dogs undergoing hindlimb orthopaedic surgery

\begin{tabular}{|c|c|c|c|c|c|}
\hline \multicolumn{6}{|c|}{ Search Outcome } \\
\hline Database & $\begin{array}{l}\text { Number } \\
\text { of results }\end{array}$ & $\begin{array}{l}\text { Excluded - } \\
\text { irrelevant to } \\
\text { the PICO } \\
\text { question }\end{array}$ & $\begin{array}{c}\text { Excluded - review } \\
\text { article }\end{array}$ & $\begin{array}{l}\text { Excluded - book } \\
\text { chapters or } \\
\text { sections, other non- } \\
\text { peer reviewed } \\
\text { material }\end{array}$ & $\begin{array}{c}\text { Total } \\
\text { relevant } \\
\text { papers }\end{array}$ \\
\hline CAB Abstracts & 36 & 33 & 0 & 2 & 1 \\
\hline Medline & 28 & 26 & 1 & 0 & 1 \\
\hline \multicolumn{5}{|c|}{ Total relevant papers when duplicates removed } & 1 \\
\hline
\end{tabular}




\section{CONFLICT OF INTEREST}

The author declares no conflicts of interest.

This Knowledge Summary was originally prepared as submission to the University of Liverpool's Evidence Based Veterinary Medicine postgraduate certificate module and thanks must go to the assessors, whose feedback has been immensely helpful in preparing this paper.

Dr Gavin Jarvis of the Department of Physiology, Development and Neuroscience at the University of Cambridge provided very useful advice on the review of this paper and I am grateful for his assistance.

\section{REFERENCES}

1. Bergh, M. S., Sullivan, C., Ferrell, C.L., Troy, J. \& Budsberg, S.C. (2014). 'Systematic review of surgical treatments for cranial cruciate ligament disease in dogs', Journal of the American Animal Hospital Association, 50(5), pp. 315-321. DOI: https://doi.org/10.5326/JAAHA-MS-6356

2. Epstein, M. E., Rodan, I., Griffenhagen, G., Kadrlik, J., Petty, M.C., Robertson, S.A. \& Simpson, W. (2015). '2015 AAHA/AAFP pain management guidelines for dogs and cats.', Journal of Feline Medicine and Surgery. SAGE Publications, 17(3), pp. 251-272. DOI: https://doi.org/10.1177/1098612X15572062

3. Heard, D. J., Webb, A. I. \& Daniels, R. T. (1986). 'Effect of acepromazine on the anesthetic requirement of halothane in the dog', American Journal of Veterinary Research, 47(10), pp. 2113-2115.

4. Reid, J., Nolan, A.M., Hughes, J.M.L., Lascelles, D., Pawson, P. \& Scott, E.M. (2007). 'Development of the short-form Glasgow Composite Measure Pain Scale (CMPS-SF) and derivation of an analgesic intervention score', Animal Welfare. Universities Federation for Animal Welfare, 16(SUPPL.), pp. 97104.

5. Smith, L. J. \& Yu, J. K. A. (2001). 'A comparison of epidural buprenorphine with epidural morphine for postoperative analgesia following stifle surgery in dogs', Veterinary Anaesthesia and Analgesia, 28(2), pp. 87-96. DOI: https://doi.org/10.1046/j.1467-2987.2000.00038.x

6. Valverde, A., Dyson, D. H. \& McDonell, W. N. (1989). 'Epidural morphine reduces halothane MAC in the dog', Canadian Journal of Anaesthesia. Springer, 36(6), pp. 629-632.

DOI: https://doi.org/10.1007/BF03005412 


\section{EVIIDEFeE

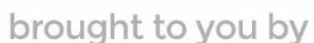 \\ RCVS KNOWLEDGE}

\section{Intellectual Property Rights}

Authors of Knowledge Summaries submitted to RCVS Knowledge for publication will retain copyright in their work, and will be required to grant RCVS Knowledge a non-exclusive license of the rights of copyright in the materials including but not limited to the right to publish, re-

publish, transmit, sell, distribute and otherwise use the materials in all languages and all media throughout the world, and to license or permit others to do so.

\section{Disclaimer}

Knowledge Summaries are a peer-reviewed article type which aims to answer a clinical question based on the best available current evidence. It does not override the responsibility

of the practitioner. Informed decisions should be made by considering such factors as individual clinical expertise and judgement along with patient's circumstances and owners' values. Knowledge Summaries are a resource to help inform and any opinions expressed within the Knowledge Summaries are the author's own and do not necessarily reflect the view of the RCVS Knowledge. Authors are responsible for the accuracy of the content. While the

Editor and Publisher believe that all content herein are in accord with current recommendations and practice at the time of publication, they accept no legal responsibility

for any errors or omissions, and make no warranty, express or implied, with respect to material contained within.

For further information please refer to our Terms of Use.

RCVS Knowledge is the independent charity associated with the Royal College of Veterinary Surgeons (RCVS). Our ambition is to become a global intermediary for evidence based veterinary knowledge by providing access to information

that is of immediate value to practicing veterinary professionals and directly contributes to evidence based clinical decision-making.

https://www.veterinaryevidence.org/

RCVS Knowledge is a registered Charity No. 230886.

Registered as a Company limited by guarantee in England and Wales No. 598443.

Registered Office: Belgravia House, 62-64 Horseferry Road, London SW1P 2AF

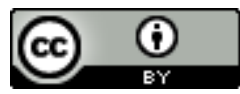

This work is licensed under a Creative Commons Attribution 4.0 International License 